\title{
In-situ SEM and TEM Nanomechanical Study of Wear and Failure Mechanisms
}

\author{
Sanjit Bhowmick ${ }^{1}$, Eric Hintsala ${ }^{1}$, Douglas Stauffer ${ }^{1}$, S.A. Syed Asif ${ }^{1}$ \\ ${ }^{1 .}$ Bruker Nano Surfaces, Eden Prairie, USA.
}

New loading modes for in situ TEM nanomechanical testing can be achieved through operation of a 2D transducer (with normal and lateral axes) to study combined normal and shear stress states during scratch testing. This allows for in situ study of friction, wear and failure mechanisms at the nanoscale under shear loading conditions. One application is visualization of film debonding processes, such as the complex industrial problem of failure of hard disc drive (HDD) multilayer film stacks. These films are highly engineered and sensitive to small plastic reorientations of the magnetic recording layer which can lead to device failure through loss of the recorded magnetic moment [1]. A second, more fundamental application is wear mechanisms of olivine single crystals. In olivine and some other ceramic materials, dislocation nucleation can contribute to frictional energy dissipation and the dependence of interfacial shear strength on dislocation density and the shearing conditions such as directionality, rate and force is of interest [2]. Olivine is commonly used as a model material for geological processes due to its abundance in the Earth's crust and nanoscale study of wear mechanisms may play an important role in developing multiscale mechanical models for fault formation.

Functional HDD multilayer films were deposited on a silicon wedge substrate by anonymous collaborators in the hard disc drive industry. This elevates the region of interest to prevent shadowing and enables a perpendicular wedge-on-wedge scratch experiment [3]. The film structure consists of a $2 \mathrm{~nm}$ diamond-like carbon film protecting two functional layers below, a metallic recording layer and its stoichiometrically equivalent oxide. For the olivine specimens, bulk olivine single crystals were wedge polished to $10 \mu \mathrm{m}$ thickness using conventional techniques. These crystals were then mounted and a $2 \mathrm{~nm}$ conductive Pt film deposited prior to final thinning by focused ion beam (FIB) machining to $\sim 100 \mathrm{~nm}$.

Both specimens were tested in situ utilized a Hysitron PI-95 PicoIndenter (Bruker Nano Surfaces, USA) equipped with a 2D MEMS transducer and sharp wedge tip with a radius of curvature $\sim 20 \mathrm{~nm}$. Constant normal load control was utilized, with an open-loop piezo actuator for the lateral axis, set for a nominal $1 \mu \mathrm{m}$ scratch length. For the HDD, normal loads ranged from 1-20 $\mu \mathrm{N}$ to explore depth dependence of the mechanical response and thereby probe different layers of the multilayer structure, while for the olivine crystal, much higher loads of $100 \mu \mathrm{N}$ were required to produce deformation, which was repeated across multiple scratching passes.

For the HDD specimens, a transition in behaviour occurred with increasing normal load. At low loads, the DLC protective layer was removed and asperities of the columnar grain tops flattened through stick slip motion as shown in Fig. 1a. At increased normal load, past $5 \mu \mathrm{N}$, plastic deformation in the functional device layers occurred, culminating in debonding between the metallic and oxide magnetic layers at $20 \mu \mathrm{N}$ as shown in Fig. 2b. Here, lateral force continues to increase throughout the test due to ploughing of the tip.

In the case of repeated scratches on the Olivine specimen, substantial dislocation plasticity was observed as shown in Fig. 2. Each pass was observed to nucleate more dislocations which were arranged in a symmetric array along the wear path. During these wear passes, the kinetic friction coefficient remained 
relatively constant near 0.1 , but the dependence of rate, force magnitude, and directionality (reciprocating, rather than one directional) will be explored in future experiments.

\section{References:}

[1] S. C. Lee et al, J. Tribol. 131 (2009), 011904.

[2] M. Mishra and I. Szlufarska, J. Mater. Sci. 48 (2013), p. 1593.

[3] E. D. Hintsala et al, JOM 69 (2017), p. 51-56.
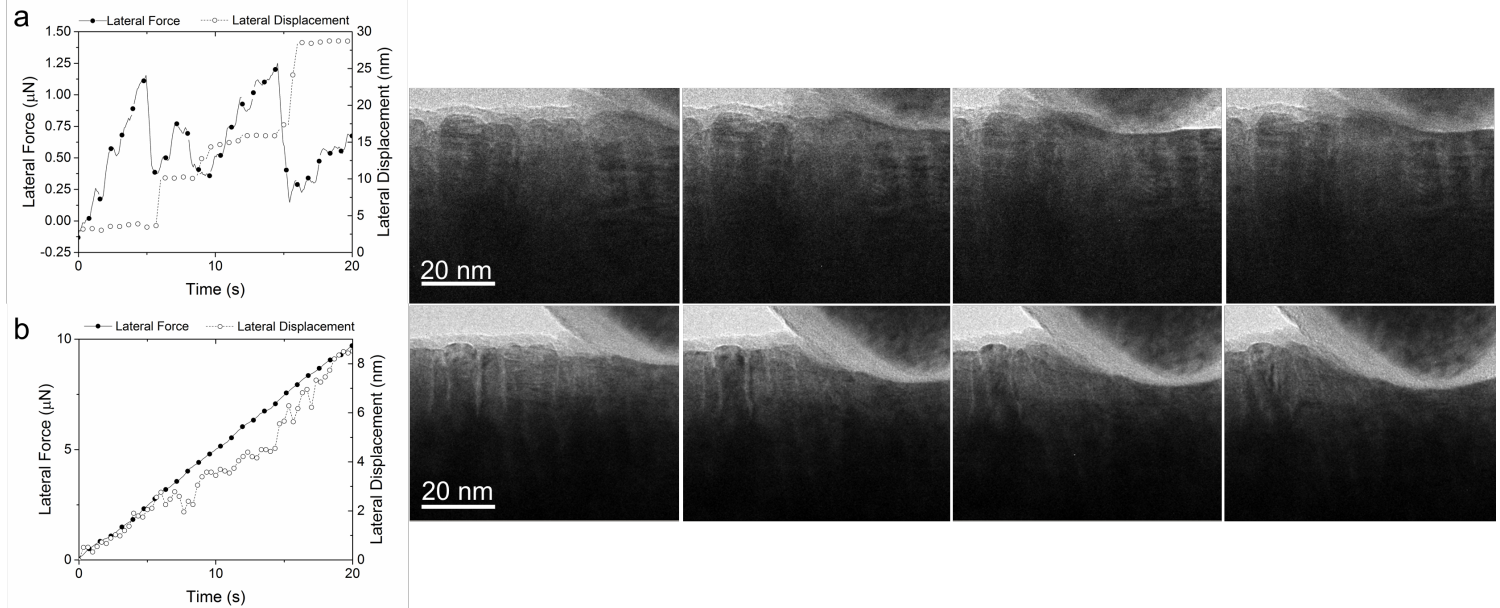

Figure 1. a) Lateral force and displacement versus time with corresponding in situ TEM images for a normal load of $2 \mu \mathrm{N}$. b) Lateral force and displacement versus time with corresponding in situ TEM images for a normal load of $20 \mu \mathrm{N}$.

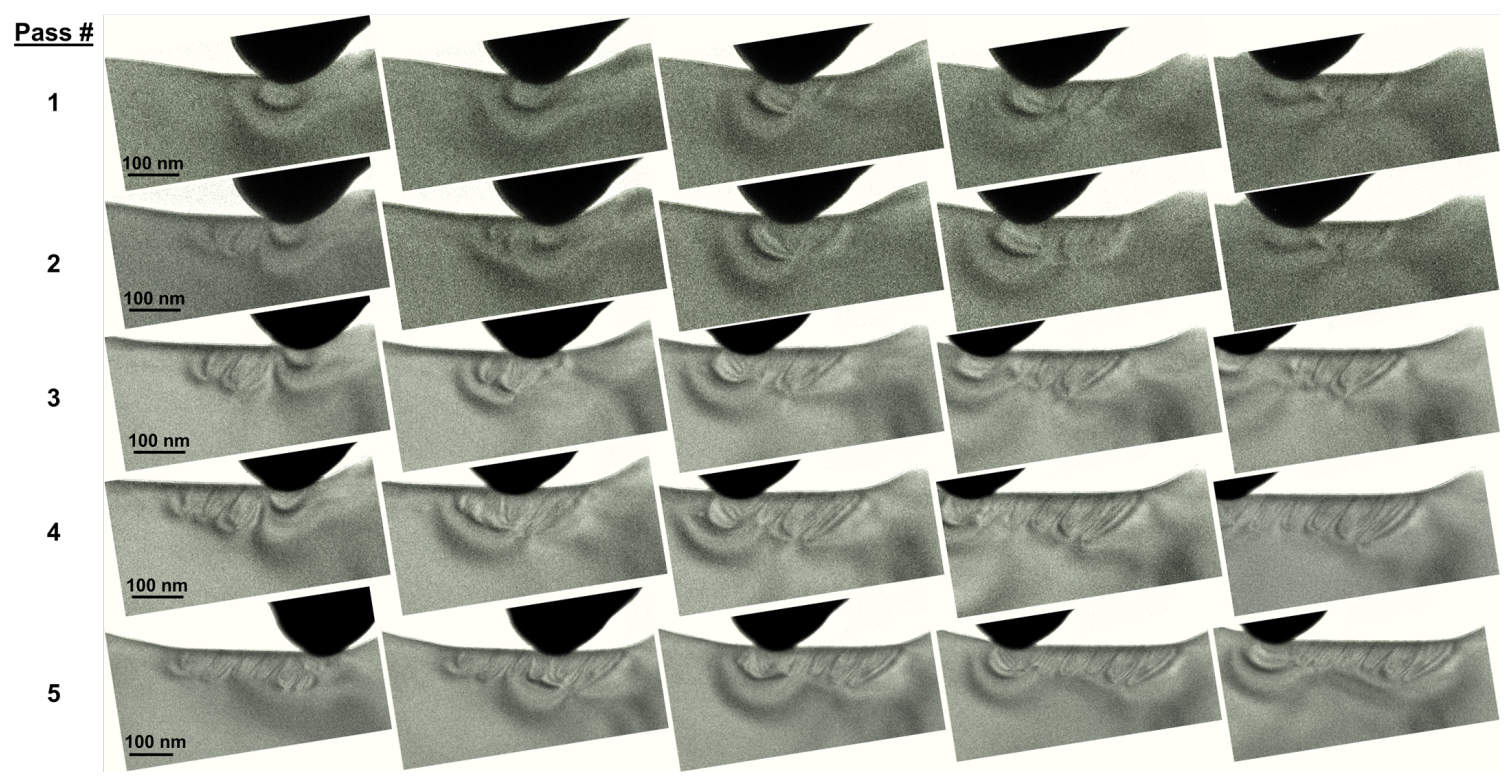

Figure 2. Image montage showing gradual dislocation nucleation during 5 passes of scratch test on single crystal olivine 